\title{
Cardiovascular Risk Prediction in Patients With Human Immunodeficiency Virus-Reply.
}

\author{
Matthew J. Feinstein, MD' ${ }^{1}$, Joseph A. Delaney, $\mathrm{PhD}^{2}$, and Heidi M. Crane, $\mathbf{M D}^{3}$ \\ ${ }^{1}$ Division of Cardiology, Department of Medicine, Northwestern University Feinberg School of \\ Medicine, Chicago, IL USA \\ ${ }^{2}$ Department of Epidemiology, School of Public Health, University of Washington, Seattle, WA \\ USA \\ ${ }^{3}$ Division of Infectious Diseases, Department of Medicine, University of Washington School of \\ Medicine, Seattle, WA USA
}

\begin{abstract}
We appreciate the interest and thoughtful comments by Raggi et al regarding our article in which we analyzed the performance of the ACC/AHA Pooled Cohort Equations (PCEs) and a de novo HIV-specific model for predicting MI among 19,829 HIV-infected (HIV+) persons in a multi-center cohort. ${ }^{1}$ Using rigorous methods ${ }^{2}$ to evaluate model performance, we found that the PCEs performed reasonably well among white HIV+ men, with calibration and discrimination generally similar to that found among uninfected cohorts. However, the PCEs performed substantially worse among HIV+ white women, black men, and black women; the HIV-specific model performed even worse. In a recent analysis ${ }^{3}$, Raggi et al. used net reclassification improvement (NRI) to evaluate three risk estimation models (one of which is HIV-specific) in a single-center Italian cohort of $2550 \mathrm{HIV}+$ patients. While there are several differences between the cohorts and methods used in our respective analyses, we both found that the Pooled Cohort Equations performed modestly among HIV+ persons and models incorporating HIV-specific variables performed no better.
\end{abstract}

As we discuss and Raggi et al. also mention, disparate rates of type II MI across race-sex groups may account for some of the differential performance of the PCEs across race-sex groups in our study. Furthermore, regardless of race-sex group, type II MI rates are elevated in HIV because sepsis, hypovolemia, and other critical illness-associated states common in HIV can lead to type II MI. Accordingly, Raggi et al. argue that (1) it may be inappropriate to apply models derived in the general population to predict primarily atherosclerotic MIs to HIV+ persons, and (2) studies of risk prediction models in HIV should distinguish type I and type II MIs.

We differ from Raggi et al. in their comment that it is inappropriate, at present, to evaluate MI risk among HIV+ persons using general population-derived risk estimators. Although it is our hope that new HIV-specific models can ultimately predict MIs among HIV+ persons more accurately than currently available models, HIV-specific models both of our studies

Corresponding Author: Matthew J. Feinstein, MD, Northwestern University Feinberg School of Medicine, 680 N. Lakeshore Drive, Suite 1400, Chicago, IL 60611, matthewjfeinstein@northwestern.edu, Phone: 847.624.2996, Fax: 312.908.9588. 
failed to produce improvements over the PCEs. Furthermore, risk estimators like the PCEs may actually perform better when used to predict only type I MIs for HIV+ persons.

We certainly agree, however, that it would be valuable for future studies in HIV+ populations to distinguish between type I and type II MIs. This is important for model fidelity and also for practical clinical reasons: strategies to prevent and treat type I MI are fairly well-established, but this is not the case for type II MIs given their diverse underlying causes. Whereas statin therapy is clearly beneficial for persons at sufficient risk for (or recurrence of) type I MIs, the role of statins in preventing and treating type II MIs is less clear. For these reasons, CNICS implemented an adjudication process that distinguishes type I and type II MIs. Ultimately, differentiating among MI types in HIV may improve the ability of risk estimation models to do what they are truly intended to do - inform preventive and therapeutic strategies that improve public health.

\section{REFERENCES}

1. Feinstein MJ, Nance RM, Drozd DR, et al. Assessing and refining myocardial infarction risk estimation among patients with human immunodeficiency virus: A study by the centers for AIDS research network of integrated clinical systems. JAMA Cardiol 20161221 Doi: 10.1001/ jamacardio.2016.4494 [Epub ahead of print]

2. Van Calster B, Nieboer D, Vergouwe Y, De Cock B, Pencina MJ, Steyerberg EW. A calibration hierarchy for risk models was defined: from utopia to empirical data. J Clin Epidemiol 2016;74:167-176. [PubMed: 26772608]

3. Raggi P, De Francesco D, Manicardi M, et al. Prediction of hard cardiovascular events in HIV patients. J Antimicrob Chemother 2016;71:3515-3518. [PubMed: 27591294] 